Mini-Review

\title{
Signaling Networks in Neuronal Polarization
}

\author{
Takeshi Yoshimura, ${ }^{1,2}$ Nariko Arimura, ${ }^{2}$ and Kozo Kaibuchi ${ }^{2}$ \\ ${ }^{1}$ Department of Molecular Biology, Graduate School of Science, Institute for Advanced Research, Nagoya University, Aichi 464-8602, Japan, and \\ ${ }^{2}$ Department of Cell Pharmacology, Graduate School of Medicine, Nagoya University, Aichi 466-8550, Japan
}

\begin{abstract}
A mature neuron is typically polarized both structurally and functionally, with a single long axon and several dendrites. Neuronal polarity is essential for unidirectional signal flow from somata or dendrites to axons. The initial event in establishing a polarized neuron is the specification of a single axon. Early in neuronal development, one immature neurite becomes differentiated from other neurites to form an axon. Although studies in the past two decades have yielded a catalog of structural, molecular, and functional differences between axons and dendrites, we are only now beginning to understand the molecular mechanisms involved in the establishment of neuronal polarity.

In the last few years, neuronal polarity-regulating molecules have been revealed. There are two major signaling cascades in neuronal polarization. Several groups, including ours, reported that the phosphatidylinositol 3-kinase (PI3-kinase)/Akt/glycogen synthase kinase-3 $\beta$ (GSK-3 $\beta$ )/collapsin response mediator protein-2 pathway is important for axon specification and elongation. Recent studies have revealed that the positive feedback loop composed of Rho family small GTPases and the Par3/Par6/atypical protein kinase C complex plays a role in the initial events of neuronal polarization downstream of PI3-kinase. Here, we discuss the roles of signaling molecules for axon specification.
\end{abstract}

Key words: axon; neuronal polarity; PI3-kinase; GSK-3 $\beta$; CRMP-2; Par complex

\section{Processes of neuronal polarization}

The ability of neuronal cells to polarize is essential for organization of the nervous system. A model system for studying neuronal polarity, cultured hippocampal neurons, was pioneered by Craig and Banker $(1994)>20$ years ago. Cultured hippocampal neurons develop a single long axon and several shorter dendrites, which maintain their structural characteristics at the molecular level. During maturation, hippocampal neurons dramatically change their morphology. Dotti et al. (1988) precisely observed this differentiation process and divided the morphological events into five stages (Fig. 1). Shortly after attachment to the substratum, a neuron extends lamellipodia (stage 1). These protrusions then develop into several short immature neurites (stage 2). At this stage, neurons still appear to be unpolarized. All neurites alternate phases of elongation and retraction and are approximately equal in length. It is difficult to determine which neurite will become an axon. Next, one of the immature neurites rapidly grows into a long neurite, which soon acquires axonal characteristics (stage 3). A few days after the formation of the axon, the remaining neurites slowly elongate to become dendrites (stage 4). Typically, the axon and dendrites continue to ma-

Received Sept. 2, 2006; revised Sept. 19, 2006; accepted Sept. 19, 2006.

This work was supported by grants-in-aid from the Ministry of Education, Culture, Sports, Science, and Technology of Japan for Creative Scientific Research, Scientific Research on Priority Areas, and Center of Excellence Research and by a grant-in-aid for Japan Society for the Promotion of Science (JSPS) Fellows from JSPS.

Correspondence should be addressed to Kozo Kaibuchi, Department of Cell Pharmacology, Graduate School of Medicine, Nagoya University, 65 Tsurumai, Showa-ku, Nagoya, Aichi 466-8550, Japan. E-mail: kaibuchi@ med.nagoya-u.ac.jp.

D01:10.1523/JNEUROSCI.3824-06.2006

Copyright $\odot 2006$ Society for Neuroscience $\quad$ 0270-6474/06/2610626-05\$15.00/0 ture and subsequently develop by $7 \mathrm{~d}$ after plating. Cultured neurons form synaptic contacts and establish a neuronal network (stage 5).

Neuronal polarization occurs from stage 2 to stage 3 . The first step in neuronal polarization is initial axon formation. The specification of the axon is thought to depend on its length relative to the other minor processes (Bradke and Dotti, 2000). Intracellular mechanisms that help to enhance neurite and axon outgrowth evidently require reorganization of cytoskeletons, including actin filaments and microtubules (Fukata et al., 2002a). A highly dynamic area is located at the tips of growing axons, where drastic rearrangements of actin filaments and microtubules occur during neurite elongation (Bradke and Dotti, 2000; Baas and Buster, 2004). Actin instability is higher in one of neurite growth cones of unpolarized neurons, and application of the actindepolymerizing drug cytochalasin D to stage 2 neurons causes multiple axon-like neurites, implying that reorganization of actin filaments is necessary for axon formation (Bradke and Dotti, 1999). Actin disassembly by cytochalasin B allows microtubules to extend distally into the peripheral region of the growth cones and leads to rapid neurite growth (Forscher and Smith, 1988). Microtubule assembly occurs in the cell body and the growth cone (Brown et al., 1992, 1993). The microtubule array in the neurite or axon is formed through two mechanisms: the transport of microtubule polymer and microtubule assembly at the plus ends of the microtubules. Both mechanisms appear to contribute to axon outgrowth (Baas, 1997). These findings suggest that actin filaments restrict the protrusion of microtubules. Dynamic actin filaments appear to allow the enhanced polymerization and/or transport of microtubules, 


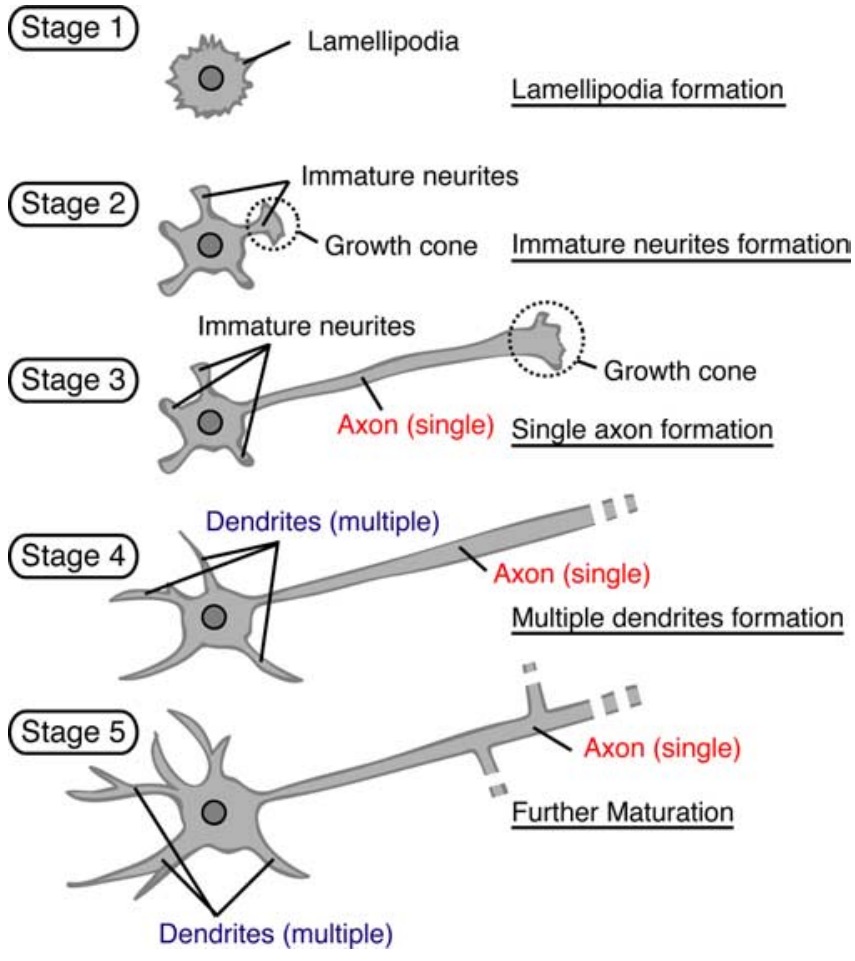

Figure 1. Processes of neuronal polarization in cultured hippocampal neurons. Cultured pyramidal neurons from a rodent hippocampus acquire their characteristic polarized morphology in five well defined stages. [Reproduced with permission from Dotti et al. (1988).]

resulting in promoted neurite elongation, followed by axon formation (Bradke and Dotti, 2000).

\section{PI3-kinase/Akt/GSK-3 $\beta$ pathway}

It is speculated that axonal fate is intrinsically specified in cultured hippocampal neurons, because cultured neurons are in a uniform environment (Craig and Banker, 1994). Conversely, it is conceivable that, in vivo, extracellular cues play a pivotal role in the specification of axonal fate. Little is known about extracellular cues that govern neuronal polarity in vivo. Some studies have shown that extracellular substrate molecules can govern which neurite becomes an axon depending on the substrate preference of neurite elongation (Esch et al., 1999). When neurons are cultured on substrates patterned with stripes of poly-L-lysine and either laminin or neuron-glia cell adhesion molecule (NgCAM), undifferentiated immature neurites attach on both substrates equally, but axons form preferentially on laminin or NgCAM. These observations suggest that the signals produced by the contact of laminin or NgCAM with adhesion molecules such as integrins cause the rapid neurite growth and are sufficient to induce axon formation. This rapid axon formation is also observed when an immature neurite in stage 2 neurons comes in contact with laminin-coated beads (Menager et al., 2004). Thus, signaling cascades accelerated by the extracellular matrix may initiate neurite growth and axon formation, and certain extracellular cues may determine axon or dendrite fate during physiological development.

Recent studies have shown the importance of PI3-kinase and its lipid product phosphatidylinositol 3,4,5-triphosphate $\left(\mathrm{PIP}_{3}\right)$ in determining and maintaining internal polarity in neurotrophils and dictyostelium (Iijima et al., 2002). Several groups, including ours, reported that local activation of PI3kinase and accumulation of $\mathrm{PIP}_{3}$ at the tip of one of the immature neurites are important for axon specification and



Figure 2. Signaling cascades in axon specification. In one immature neurite (the future axon), the extracellular matrix (ECM) activates PI3-kinase through interaction with adhesion molecules or receptors, thereby producing $\mathrm{PIP}_{3}$. Accumulated $\mathrm{PIP}_{3}$ drives two major signaling cascades: the Akt/GSK-3 $\beta /$ CRMP-2 pathway and the positive feedback loop composed of Cdc42, the Par complex, and Rac1. These signaling cascades regulate cytoskeletons, endocytosis, protein trafficking, and transcriptions to promote neurite elongation and to determine axon or dendrite fate.

elongation (Shi et al., 2003; Menager et al., 2004) (Fig. 2). PI3-kinase inhibitors, such as LY294002 [2-(4-morpholinyl)8-phenyl-4H-1-benzopyran-4-one], delay the transition of neurons from stage 1 to stage 3 , affecting both axon formation and elongation (Shi et al., 2003; Menager et al., 2004). Local contact of immature neurites with the extracellular matrix, such as laminin, induces rapid production of $\mathrm{PIP}_{3}$ at the tip of the neurite through the action of PI3-kinase, and PIP 3 is involved in axon specification, possibly by stimulating elongation of an immature neurite (Menager et al., 2004). We reported previously that $\mathrm{PIP}_{3}$ seems to be transported toward the contact site (Menager et al., 2004). In fact, Horiguchi et al. (2006) recently demonstrated that a complex of guanylate kinase-associated kinesin and a $\mathrm{PIP}_{3}$-interacting protein, $\mathrm{PIP}_{3} \mathrm{BP}$, transports $\mathrm{PIP}_{3}$ to the neurite end. The phosphatase and tensin homolog deleted on chromosome 10 (PTEN) is a lipid and protein phosphatase that functions in an opposite manner from PI3-kinase by dephosphorylating PIP $_{3}$ (Maehama and Dixon, 1998). Overexpression of PTEN inhibits axon formation, whereas knockdown of PTEN by short interfering RNA ( siRNA) induces formation of multiple axons (Jiang et al., 2005).

PI3-kinase activates Akt (also called protein kinase B) by phosphorylation of Akt at Thr-308 and Ser-473 via PIP $_{3}$, phosphoinositide-dependent kinase, and integrin-linked kinase (ILK) (Scheid and Woodgett, 2001; Hannigan et al., 2005). Constitutively active myristoylated Akt leads to formation of multiple axons (Jiang et al., 2005). GSK-3 $\beta$ is known to be constitutively active. Activated Akt and ILK phosphorylate GSK-3 $\beta$ at Ser-9 and inactivate its kinase activity (Grimes and Jope, 2001; Hannigan et al., 2005). GSK-3 $\beta$ relays signals from PTEN and Akt, and the decreased activity of GSK-3 $\beta$ is required for neuronal polarization (Jiang et al., 2005; Yoshimura et al., 2005). Inhibition of GSK-3 $\beta$ by GSK-3 inhibitors [ $\mathrm{LiCl}$ (lithium chloride), SB216763, and SB415286] or hairpin siRNA induces formation of multiple axons, whereas overexpression of constitutively active GSK-3 $\beta$ 
inhibits axon formation (Jiang et al., 2005; Yoshimura et al., 2005).

Recently, it was shown that local Akt degradation mediated by the ubiquitin-proteasome system is important in determining neuronal polarity (Yan et al., 2006). Akt is present in both the cell body and multiple immature neurites of stage 2 neurons. Preferential degradation of putative future dendritic Akt is mediated by the ubiquitin-proteasome system from stage 2 to stage 3 ; and in stage 3 neurons, Akt is localized in the cell body and one axonal tip. There is more inactivated and phosphorylated GSK-3 $\beta$ at Ser-9 in the tips of axons than in the tips of future dendrites in stage 3 neurons (Jiang et al., 2005). At the tips of nascent axons, Akt phosphorylates and inactivates GSK-3 $\beta$. These results indicate the significance of the PI3-kinase/Akt/GSK-3 $\beta$ pathway in neuronal polarity (Fig. 2).

\section{Regulation of collapsin response mediator protein-2 by GSK-3 $\beta$}

Collapsin response mediator protein-2 (CRMP-2) is one of at least five isoforms and is highly and exclusively expressed in the developing nervous system (Goshima et al., 1995; Wang and Strittmatter, 1996; Arimura et al., 2004). Mutations in the UNC-33 gene, a Caenorhabditis elegans homolog of CRMPs, lead to severely uncoordinated movement and abnormalities in the guidance of axons of many neurons (Hedgecock et al., 1985). We showed that GSK-3 $\beta$ determines axon or dendrite fate through phosphorylation of CRMP-2 (Yoshimura et al., 2005). CRMP-2 is enriched in the growing axon of hippocampal neurons, and overexpression of CRMP-2 induces multiple axons, whereas inhibition of CRMP-2 functions impairs axon formation (Inagaki et al., 2001). CRMP-2 interacts with tubulin heterodimers and promotes microtubule assembly in vitro (Fukata et al., 2002b). CRMP-2 also regulates endocytosis of specific adhesion molecules, including L1, through interaction with Numb (Nishimura et al., 2003) and reorganization of actin filaments acting through specifically Rac1-associated protein 1 (Sra-1) (Kawano et al., 2005). Truncated kinesin-1 selectively accumulates in only (future) axons in the early and late stages (Nakata and Hirokawa, 2003; Jacobson et al., 2006). CRMP-2 links tubulin heterodimers or Sra-1 to kinesin-1 through interaction with kinesin light chain, and the CRMP$2 /$ kinesin- 1 complex regulates the transport of these proteins to the distal part of the growing axon (Kawano et al., 2005; Kimura et al., 2005). Thus, CRMP-2 seems to promote neurite elongation and axon specification by regulating microtubule assembly, endocytosis of adhesion molecules, reorganization of actin filaments, and axonal protein trafficking.

GSK-3 $\beta$ can phosphorylate CRMP-2 at Thr-514 and Ser518 through the priming phosphorylation at Ser- 522 by Cdk 5 (Yoshimura et al., 2005). Phosphorylation of CRMP-2 by GSK-3 $\beta$ lowers the activity of CRMP-2 for the interaction with tubulin and Numb (Arimura et al., 2005; Yoshimura et al., 2005). In cultured hippocampal neurons, $\sim 30 \%$ of CRMP- 2 is constitutively phosphorylated at Thr-514, and this phosphorylation is decreased by GSK-3 inhibitors. GSK-3 $\beta$ is activated only in the tips of axons in stage 3 neurons (Jiang et al., 2005). CRMP-2 phosphorylated at Thr-514 is enriched in the distal part of the growing axons but clearly not at the axonal growth cones, suggesting that there is a nonphosphorylated CRMP-2 pool at Thr-514 in the growing axonal growth cone (Yoshimura et al., 2005). Expression of constitutively active GSK-3 $\beta$ impaired neuronal polarization, whereas the non- phosphorylated form of CRMP-2 counteracted the inhibitory effects of GSK- $3 \beta$, which indicates that GSK- $3 \beta$ regulates neuronal polarity through phosphorylation of CRMP-2 (Yoshimura et al., 2005) (Fig. 2).

Zhou et al. (2004) reported that nerve growth factorinduced axon elongation is mediated by the PI3-kinase/ILK/ GSK-3 $\beta$ /adenomatous polyposis coli (APC) pathway in dorsal root ganglion neurons. GSK-3 $\beta$ phosphorylates microtubuleassociated protein 1B (MAP1B) and APC (Goold et al., 1999; Zumbrunn et al., 2001; Trivedi et al., 2005). Phosphorylation of MAP1B by GSK- $3 \beta$ suppresses detyrosination of microtubules and decreases the number of stable microtubules. Binding of APC to microtubules increases microtubule stability, whereas phosphorylation of APC decreases interaction with microtubules. Hippocampal neurons derived from doubleknock-out mice with disrupted tau and MAP1B genes, which have redundant functions, show a defect in axon formation at stage 3 (Takei et al., 2000). These results suggest that GSK-3 $\beta$ regulates microtubule dynamics through microtubule-associating molecules and thereby governs neuronal polarity.

\section{Positive feedback loop}

The Par complex (including Par3, Par6, and atypical protein kinase C) functions in various cell-polarization events, including axon specification (Ohno, 2001; Shi et al., 2003; Macara, 2004; Nishimura et al., 2004). Rho family small GTPases are major regulators of actin filaments and microtubules (Govek et al., 2005). Rho family small GTPases cycle between a GTP-bound active state and a GDP-bound inactive state, acting as molecular switches. Guanine nucleotide exchange factors (GEFs) activate GTPases by enhancing the exchange of bound GDP for GTP (Schmidt and Hall, 2002). Of the Rho family small GTPases, Cdc42, Racl, and RhoA have been characterized most extensively. PI3-kinase activity is also required for proper localization of the Par complex and Cdc42 at the tips of the growing axons, all of which are necessary for neuronal polarization (Shi et al., 2003; Schwamborn and Puschel, 2004). Nishimura et al. (2004) reported that Par3 is transported to the distal tip of the growing axon by kinesin- 2 through direct interaction with KIF3A and that proper localization of Par3 is required to establish neuronal polarity. T-lymphoma invasion and metastasis 1 (Tiam1; a GEF for Rac1) is involved in axon formation (Kunda et al., 2001). Cdc42GTP binds to Par6 and determines the localization of the Par complex. Par3 directly interacts with STEF (Sif- and Tiam1-like exchange factor)/Tiam1 (GEFs for Rac1), and the Par3/Par6 complex mediates the signal from Cdc42 to Rac1 for axon specification (Nishimura et al., 2005). Given that Rac1 activates PI3kinase, the signal initially evoked by PI3-kinase appears to terminate at PI3-kinase itself (Govek et al., 2005). This positive feedback loop may be a driving force for axon specification and maturation (Fig. 2).

It has been reported that Rap1B and $\mathrm{H}$-Ras, members of the Ras family small GTPases, are involved in axon specification (Schwamborn and Puschel, 2004; Yoshimura et al., 2006). Rap1B acts upstream of $\mathrm{Cdc} 42$ and the Par complex in neuronal polarity (Schwamborn and Puschel, 2004). Analysis using inhibitors revealed that PI3-kinase functions upstream of Rap1B. What is the upstream signaling molecule of PI3-kinase on neuronal polarity? We recently found that $\mathrm{H}$-Ras plays critical roles in establishing neuronal polarity upstream of the PI3-kinase/Akt/GSK-3 $\beta$ / CRMP-2 pathway (Yoshimura et al., 2006). Ras may stimulate and activate PI3-kinase at the tip of one of the immature neurites downstream of the extracellular cues (Fig. 2). 


\section{Concluding remarks}

Significant progress has been made toward understanding the intracellular signaling cascades during neuronal polarization. Figure 2 is a model schema of the signaling cascades in axon specification. Two major signaling cascades downstream of PI3kinase play a central role in neuronal polarization. The PI3kinase/Akt.GSK-3 $\beta /$ CRMP-2 pathway promotes neurite outgrowth to determine axon or dendrite fate. The positive feedback loop composed of Cdc42, the Par complex, and Racl cycles locally in the tips of future axons. However, more questions must be answered before the molecular mechanisms will be entirely understood. What are the extracellular signals involved in the establishment of neuronal polarity? Because cultured neurons acquire polarity without any directional gradients of extrinsic signals, neurons appear to have an internal polarization program (Craig and Banker, 1994). However, axons and dendrites face the preferred direction in vivo, where extrinsic signals from the surrounding cellular environment likely play a major role in neuronal polarization. It remains primarily unknown about extracellular cues that govern neuronal polarity. Additional studies are needed to elucidate fully the mechanisms behind establishment of neuronal polarity.

In mature regenerating dorsal root ganglion neurons, inactivation of GSK- $3 \beta$ and accumulation of APC were observed at the distal tips of axons (Zhou et al., 2004). Overexpression of CRMP-2 in injured rat hypoglossal motor neurons accelerates nerve regeneration (Suzuki et al., 2003). It would be interesting to examine what kinds of signaling cascades are involved in nerve regeneration. Investigating the details of the signaling would yield a more complete understanding of a diverse range of processes including development, nerve regeneration, and neurodegenerative disease.

\section{References}

Arimura N, Menager C, Fukata Y, Kaibuchi K (2004) Role of CRMP-2 in neuronal polarity. J Neurobiol 58:34-47.

Arimura N, Menager C, Kawano Y, Yoshimura T, Kawabata S, Hattori A, Fukata Y, Amano M, Goshima Y, Inagaki M, Morone N, Usukura J, Kaibuchi K (2005) Phosphorylation by Rho kinase regulates CRMP-2 activity in growth cones. Mol Cell Biol 25:9973-9984.

Baas PW (1997) Microtubules and axonal growth. Curr Opin Cell Biol 9:29-36.

Baas PW, Buster DW (2004) Slow axonal transport and the genesis of neuronal morphology. J Neurobiol 58:3-17.

Bradke F, Dotti CG (1999) The role of local actin instability in axon formation. Science 283:1931-1934.

Bradke F, Dotti CG (2000) Establishment of neuronal polarity: lessons from cultured hippocampal neurons. Curr Opin Neurobiol 10:574-581.

Brown A, Slaughter T, Black MM (1992) Newly assembled microtubules are concentrated in the proximal and distal regions of growing axons. J Cell Biol 119:867-882.

Brown A, Li Y, Slaughter T, Black MM (1993) Composite microtubules of the axon: quantitative analysis of tyrosinated and acetylated tubulin along individual axonal microtubules. J Cell Sci 104:339-352.

Craig AM, Banker G (1994) Neuronal polarity. Annu Rev Neurosci 17:267-310.

Dotti CG, Sullivan CA, Banker GA (1988) The establishment of polarity by hippocampal neurons in culture. J Neurosci 8:1454-1468.

Esch T, Lemmon V, Banker G (1999) Local presentation of substrate molecules directs axon specification by cultured hippocampal neurons. J Neurosci 19:6417-6426.

Forscher P, Smith SJ (1988) Actions of cytochalasins on the organization of actin filaments and microtubules in a neuronal growth cone. J Cell Biol 107:1505-1516.

Fukata Y, Kimura T, Kaibuchi K (2002a) Axon specification in hippocampal neurons. Neurosci Res 43:305-315.

Fukata Y, Itoh TJ, Kimura T, Menager C, Nishimura T, Shiromizu T, Wa- tanabe H, Inagaki N, Iwamatsu A, Hotani H, Kaibuchi K (2002b) CRMP-2 binds to tubulin heterodimers to promote microtubule assembly. Nat Cell Biol 4:583-591.

Goold RG, Owen R, Gordon-Weeks PR (1999) Glycogen synthase kinase $3 \beta$ phosphorylation of microtubule-associated protein $1 \mathrm{~B}$ regulates the stability of microtubules in growth cones. J Cell Sci 112:3373-3384.

Goshima Y, Nakamura F, Strittmatter P, Strittmatter SM (1995) Collapsininduced growth cone collapse mediated by an intracellular protein related to UNC-33. Nature 376:509-514.

Govek EE, Newey SE, Van Aelst L (2005) The role of the Rho GTPases in neuronal development. Genes Dev 19:1-49.

Grimes CA, Jope RS (2001) The multifaceted roles of glycogen synthase kinase $3 \beta$ in cellular signaling. Prog Neurobiol 65:391-426.

Hannigan G, Troussard AA, Dedhar S (2005) Integrin-linked kinase: a cancer therapeutic target unique among its ILK. Nat Rev Cancer 5:51-63.

Hedgecock EM, Culotti JG, Thomson JN, Perkins LA (1985) Axonal guidance mutants of Caenorhabditis elegans identified by filling sensory neurons with fluorescein dyes. Dev Biol 111:158-170.

Horiguchi K, Hanada T, Fukui Y, Chishti AH (2006) Transport of $\mathrm{PIP}_{3}$ by GAKIN, a kinesin-3 family protein, regulates neuronal cell polarity. J Cell Biol 174:425-436.

Iijima M, Huang YE, Devreotes P (2002) Temporal and spatial regulation of chemotaxis. Dev Cell 3:469-478.

Inagaki N, Chihara K, Arimura N, Menager C, Kawano Y, Matsuo N, Nishimura T, Amano M, Kaibuchi K (2001) CRMP-2 induces axons in cultured hippocampal neurons. Nat Neurosci 4:781-782.

Jacobson C, Schnapp B, Banker GA (2006) A change in the selective translocation of the kinesin-1 motor domain marks the initial specification of the axon. Neuron 49:797-804.

Jiang H, Guo W, Liang X, Rao Y (2005) Both the establishment and the maintenance of neuronal polarity require active mechanisms: critical roles of GSK-3 $\beta$ and its upstream regulators. Cell 120:123-135.

Kawano Y, Yoshimura T, Tsuboi D, Kawabata S, Kaneko-Kawano T, Shirataki H, Takenawa T, Kaibuchi K (2005) CRMP-2 is involved in kinesin-1-dependent transport of the Sra-1/WAVE1 complex and axon formation. Mol Cell Biol 25:9920-9935.

Kimura T, Arimura N, Fukata Y, Watanabe H, Iwamatsu A, Kaibuchi K (2005) Tubulin and CRMP-2 complex is transported via kinesin-1. J Neurochem 93:1371-1382.

Kunda P, Paglini G, Quiroga S, Kosik K, Caceres A (2001) Evidence for the involvement of Tiam1 in axon formation. J Neurosci 21:2361-2372.

Macara IG (2004) Parsing the polarity code. Nat Rev Mol Cell Biol 5:220-231.

Maehama T, Dixon JE (1998) The tumor suppressor, PTEN/MMAC1, dephosphorylates the lipid second messenger, phosphatidylinositol 3,4,5trisphosphate. J Biol Chem 273:13375-13378.

Menager C, Arimura N, Fukata Y, Kaibuchi K (2004) PIP $_{3}$ is involved in neuronal polarization and axon formation. J Neurochem 89:109-118.

Nakata T, Hirokawa N (2003) Microtubules provide directional cues for polarized axonal transport through interaction with kinesin motor head. J Cell Biol 162:1045-1055.

Nishimura T, Fukata Y, Kato K, Yamaguchi T, Matsuura Y, Kamiguchi H, Kaibuchi K (2003) CRMP-2 regulates polarized Numb-mediated endocytosis for axon growth. Nat Cell Biol 5:819-826.

Nishimura T, Kato K, Yamaguchi T, Fukata Y, Ohno S, Kaibuchi K (2004) Role of the PAR-3-KIF3 complex in the establishment of neuronal polarity. Nat Cell Biol 6:328-334.

Nishimura T, Yamaguchi T, Kato K, Yoshizawa M, Nabeshima Y, Ohno S, Hoshino M, Kaibuchi K (2005) PAR-6-PAR-3 mediates Cdc42-induced Rac activation through the Rac GEFs STEF/Tiam1. Nat Cell Biol 7:270-277.

Ohno S (2001) Intercellular junctions and cellular polarity: the PAR-aPKC complex, a conserved core cassette playing fundamental roles in cell polarity. Curr Opin Cell Biol 13:641-648.

Scheid MP, Woodgett JR (2001) PKB/AKT: functional insights from genetic models. Nat Rev Mol Cell Biol 2:760-768.

Schmidt A, Hall A (2002) Guanine nucleotide exchange factors for Rho GTPases: turning on the switch. Genes Dev 16:1587-1609.

Schwamborn JC, Puschel AW (2004) The sequential activity of the GTPases Rap1B and Cdc42 determines neuronal polarity. Nat Neurosci 7:923-929. 
Shi SH, Jan LY, Jan YN (2003) Hippocampal neuronal polarity specified by spatially localized $\mathrm{mPar} 3 / \mathrm{mPar6}$ and PI 3-kinase activity. Cell 112:63-75.

Suzuki Y, Nakagomi S, Namikawa K, Kiryu-Seo S, Inagaki N, Kaibuchi K, Aizawa H, Kikuchi K, Kiyama H (2003) Collapsin response mediator protein-2 accelerates axon regeneration of nerve-injured motor neurons of rat. J Neurochem 86:1042-1050.

Takei Y, Teng J, Harada A, Hirokawa N (2000) Defects in axonal elongation and neuronal migration in mice with disrupted tau and map $1 b$ genes. J Cell Biol 150:989-1000.

Trivedi N, Marsh P, Goold RG, Wood-Kaczmar A, Gordon-Weeks PR (2005) Glycogen synthase kinase- $3 \beta$ phosphorylation of MAP1B at Ser1260 and Thr1265 is spatially restricted to growing axons. J Cell Sci 118:993-1005.

Wang LH, Strittmatter SM (1996) A family of rat CRMP genes is differentially expressed in the nervous system. J Neurosci 16:6197-6207.

Yan D, Guo L, Wang Y (2006) Requirement of dendritic Akt degradation by the ubiquitin-proteasome system for neuronal polarity. J Cell Biol 174:415-424.

Yoshimura T, Kawano Y, Arimura N, Kawabata S, Kikuchi A, Kaibuchi K (2005) GSK-3 $\beta$ regulates phosphorylation of CRMP-2 and neuronal polarity. Cell 120:137-149.

Yoshimura T, Arimura N, Kawano Y, Kawabata S, Wang S, Kaibuchi K (2006) Ras regulates neuronal polarity via the PI3-kinase/Akt/GSK-3 $\beta$ / CRMP-2 pathway. Biochem Biophys Res Commun 340:62-68.

Zhou FQ, Zhou J, Dedhar S, Wu YH, Snider WD (2004) NGF-induced axon growth is mediated by localized inactivation of GSK-3 $\beta$ and functions of the microtubule plus end binding protein APC. Neuron 42:897-912.

Zumbrunn J, Kinoshita K, Hyman AA, Nathke IS (2001) Binding of the adenomatous polyposis coli protein to microtubules increases microtubule stability and is regulated by GSK3 $\beta$ phosphorylation. Curr Biol 11: $44-49$. 